\begin{tabular}{|c|c|c|}
\hline \begin{tabular}{|l|} 
PROD. TYPE: COM \\
PP:1-10 (col.fig.: nil)
\end{tabular} & JSP|2904 DTD VER: 5.0.1 & \begin{tabular}{|l|} 
ED: Bhagya \\
PAGN: VD - SCAN: nil
\end{tabular} \\
\hline \multicolumn{3}{|c|}{ ARTICLE IN PRESS } \\
\hline$x^{2}$ & $\begin{array}{l}\text { Available online at www.sciencedirect.com } \\
\text { SCIENCE DIRECT }\end{array}$ & $\begin{array}{l}\text { journal of } \\
\text { statistical planning }\end{array}$ \\
\hline ELSEVIER & $\begin{array}{l}\text { Journal of Statistical Planning and } \\
\text { Inference } \mathbf{I I}(\mathbf{I I I}) \mathbf{I I}-\mathbf{I I I}\end{array}$ & 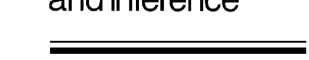 \\
\hline
\end{tabular}

\title{
Universal codes as a basis for nonparametric testing of serial independence for time series ${ }^{2}$
}

Boris Ryabko ${ }^{\mathrm{a}, *}$, Jaakko Astola $^{\mathrm{b}}$

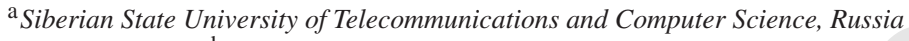

${ }^{\mathrm{b}}$ Tampere University of Technology, Finland

\section{Abstract}

We address the problem of nonparametric testing of serial independence for time series and its generalization. More precisely, we consider a stationary and ergodic source $p$, which generates symbols $x_{1} \ldots x_{t}$ from some finite set $A$ and a null hypothesis $\mathrm{H}_{0}$ that $p$ is a Markov source of order at most $m,(m \geqslant 0)$. The alternative hypothesis $\mathrm{H}_{1}$ is that the sequence is generated by a stationary and ergodic source, which differs from the source under $\mathrm{H}_{0}$. In particular, if $m=0$ we have the null hypothesis $\mathrm{H}_{0}$ that the sequence is generated by a Bernoulli source (i.e. the hypothesis that $x_{1} \ldots x_{t}$ are independent). In this paper some new tests that are based on so-called universal codes and universal predictors, are suggested.

17 (C) 2005 Elsevier B.V. All rights reserved.

MSC: 60G10; 60J10; 62M02; 62M07; 94A29

19 Keywords: Independence; Serial independence; Universal coding; Hypothesis testing; Information theory; Markov process; Random process; Prediction

\footnotetext{
Research was supported by the joint project grant "Efficient randomness testing of random and pseudorandom number generators" of Royal Society, UK (Grant ref: 15995) and Russian Foundation for Basic Research (Grant no. 03-01-00495.).

* Corresponding author.

E-mail address: boris@ ryabko.net (B. Ryabko).
} 


\section{Introduction}

Nonparametric testing of independence in time series is very important in statistical applications. There is an extensive literature dealing with nonparametric independence testing. We mention only the well-known methods that are based on the chi-square tests (see for review Kendall and Stuart, 1961) and the classical papers of Hoeffding (1948) and Blum et al. (1961); quite a full review can also be found in Ghoudi et al. (2001).

In this paper, we consider a source (or process), which generates elements from a finite set $A$ and the following two hypotheses: $\mathrm{H}_{0}$ that the source is Markovian one of order not

9 larger than $m,(m \geqslant 0)$, and the alternative hypothesis $\mathrm{H}_{1}$ that the sequence is generated by a stationary and ergodic source, which differs from the source under $\mathrm{H}_{0}$. The test should be based on a sample $x_{1} \ldots x_{t}$ generated by the source.

For example, the sequence $x_{1} \ldots x_{t}$ might be a DNA-string and one can consider the question about the depth of the statistical dependence.

We suggest a family of tests that are based on so-called universal predictors (or universal data compression methods). The Type I errors of the tests are not larger than a given $\alpha(\alpha \in$ $(0,1))$ for any source under $\mathrm{H}_{0}$, whereas the Type II error for any source under $\mathrm{H}_{1}$ tends to 0 , when the sample size $t$ grows.

The tests are based on results and ideas of Information Theory and, especially, on those of universal coding. Informally, the idea of the tests can be described as follows. Suppose that the source generates letters from an alphabet $A$ and one wants to test $\mathrm{H}_{0}$ (the source is Markovian of order $m, m \geqslant 0$ ). First we recall that there exist so-called universal codes which, loosely speaking can "compress" any sequence of length $t$ generated by a stationary and ergodic source, to the length $t h_{\infty}$ bits, where $h_{\infty}$ is the limiting Shannon entropy as $t$ tends to infinity. Secondly, it is well known in Information Theory that $h_{\infty}$ equals $m$ th-order (conditional) Shannon entropy $h_{m}$, if $\mathrm{H}_{0}$ is true, and $h_{\infty}$ is strictly less than $h_{m}$ if $\mathrm{H}_{1}$ is true. So, the following test appears natural: compress the sample sequence $x_{1} \ldots x_{t}$ by a universal code and compare the length of the obtained file with $t h_{m}^{*}$, where $h_{m}^{*}$ is an estimate of $h_{m}$. If the length of the compressed file is significantly less than $t h_{m}^{*}$, then the hypothesis $\mathrm{H}_{0}$ should be rejected.

It is no surprise that the results and ideas of universal coding can be applied to some classical problems of mathematical statistics. In fact, methods of universal coding (and the closely connected universal prediction) extract information from observed data in order to compress (or predict) data efficiently in the case where the source statistics is unknown. Recently such a connection between universal coding and mathematical statistics was used by Csiszár and Shields (2000) for estimating the order of Markov sources and by Ryabko and Monarev (2005) for constructing efficient tests for randomness, i.e. for testing the hypothesis $\hat{\mathrm{H}}_{0}$ that a sequence is generated by a Bernoulli source and all letters have equal probabilities against $\hat{\mathrm{H}}_{1}$ that the sequence is generated by a stationary and ergodic source, which differs from the source under $\hat{\mathrm{H}}_{0}$.

The outline of the paper is as follows. The next part contains definitions and necessary information from the theory of universal coding and universal prediction. Part three is devoted to testing the above described hypotheses. All proofs are given in the appendix. 


\section{2. Definitions and preliminaries}

Consider an alphabet $A=\left\{a_{1}, \ldots, a_{n}\right\}$ with $n \geqslant 2$ letters and denote by $A^{t}$ the set of words $x_{1} \cdots x_{t}$ of length $t$ from $A$. Let $p$ be a source which generates letters from $A$. Formally, $p$ is a probability distribution on the set of words of infinite length or, more simply, $p=\left(p^{t}\right)_{t} \geqslant 1$ is a consistent set of probabilities over the sets $A^{t} ; t \geqslant 1$. By $M_{\infty}(A)$ we denote the set of all stationary and ergodic sources, which generate letters from $A$. Let $M_{m}(A) \subset M_{\infty}(A)$ be the set of Markov sources of order $m, m \geqslant 0$. More precisely, $p \in M_{m}(A)$ if

$$
\begin{aligned}
& p\left(x_{t+1}=a_{i_{1}} / x_{t}=a_{i_{2}}, x_{t-1}=a_{i_{3}}, \ldots, x_{t-m+1}=a_{i_{m+1}}, \ldots\right) \\
& \quad=p\left(x_{t+1}=a_{i_{1}} / x_{t}=a_{i_{2}}, x_{t-1}=a_{i_{3}}, \ldots, x_{t-m+1}=a_{i_{m+1}}\right)
\end{aligned}
$$

9 for all $t \geqslant m$ and $a_{i_{1}}, a_{i_{2}}, \ldots \in A$. By definition, $M_{0}(A)$ is the set of all Bernoulli (or i.i.d.) sources over $A$.

\subsection{Universal prediction}

Now we briefly describe some results and methods of universal coding and prediction, which will be used later. Let a source generate a message $x_{1} \ldots x_{t-1} x_{t} \ldots$ and let $v^{t}(a)$ denote the count of letter $a$ occurring in the word $x_{1} \ldots x_{t-1} x_{t}$. After the first $t$ letters $x_{1}, \ldots, x_{t-1}, x_{t}$ have been processed the following letter $x_{t+1}$ is to be predicted. By definition, a prediction is a set of nonnegative numbers $\gamma\left(a_{1} \mid x_{1} \cdots x_{t}\right), \ldots, \gamma\left(a_{n} \mid x_{1} \cdots x_{t}\right)$ which are estimates of the unknown conditional probabilities $p\left(a_{1} \mid x_{1} \cdots x_{t}\right), \ldots, p\left(a_{n} \mid x_{1} \cdots x_{t}\right)$, i.e. of the probabilities $p\left(x_{t+1}=a_{i} \mid x_{1} \cdots x_{t}\right) ; i=1, \ldots, n$.

19 Laplace suggested the following predictor:

$$
L\left(a \mid x_{1} \cdots x_{t}\right)=\left(v^{t}(a)+1\right) /(t+|A|),
$$

where $|A|$ is the number of letters in the alphabet $A$, see Feller (1970). For example, if $A=\{0,1\}, x_{1} \ldots x_{5}=01010$, then the Laplace prediction is as follows: $L\left(x_{6}=0 \mid 01010\right)=$ $(3+1) /(5+2)=\frac{4}{7}, L\left(x_{6}=1 \mid 01010\right)=(2+1) /(5+2)=\frac{3}{7}$.

In Information Theory the error of prediction often is estimated by the Kullback-Leibler $(\mathrm{K}-\mathrm{L})$ divergence between a distribution $p$ and its estimate. Consider a source $p$ and a predictor $\gamma$. The error is characterized by the divergence

$$
\rho_{\gamma, p}\left(x_{1} \cdots x_{t}\right)=\sum_{a \in A} p\left(a \mid x_{1} \cdots x_{t}\right) \log \frac{p\left(a \mid x_{1} \cdots x_{t}\right)}{\gamma\left(a \mid x_{1} \cdots x_{t}\right)} .
$$

(Here and below $\log \equiv \log _{2}$.) It is well known that for any distributions $p$ and $\gamma$ the $\mathrm{K}-\mathrm{L}$ divergence is nonnegative and equals 0 if and only if $p(a)=\gamma(a)$ for all $a$, see, for example, Gallager (1968), that is why the $\mathrm{K}-\mathrm{L}$ divergence is a natural estimate of the prediction error. For a fixed $t, \rho_{\gamma, p}$ is a random variable, because $x_{1}, x_{2}, \ldots, x_{t}$ are random variables. We define the average error at time $t$ by

$$
\rho^{t}(p \| \gamma)=E\left(\rho_{\gamma, p}(\cdot)\right)=\sum_{x_{1} \cdots x_{t} \in A^{t}} p\left(x_{1} \cdots x_{t}\right) \rho_{\gamma, p}\left(x_{1} \cdots x_{t}\right) .
$$


1 It is known that the error of the Laplace predictor goes to 0 for any Bernoulli source $p$. More precisely, it is proven that

$$
\rho^{t}(p \| L)<(|A|-1) /(t+1)
$$

for any source $p$; Ryabko (1990) (see also Ryabko and Topsoe, 2002).

Obviously, the convergence to 0 of a predictor's error for any source from some set $M$ is an important property. For example, we can see from (3) that it is true for the Laplace predictor and the set of Bernoulli sources $M_{0}(A)$. Unfortunately, it is known that a predictor, for which error (2) goes to 0 (with probability 1 ) for any stationary and ergodic source, does not exist. More precisely, for any predictor $\gamma$ there exists a stationary and ergodic source $\tilde{p}$, such that $\lim \sup _{t \rightarrow \infty} \rho_{\gamma, \tilde{p}}\left(x_{1} \cdots x_{t}\right) \geqslant \mathrm{const}>0$ with probability 1 ; Ryabko (1988). (See also Algoet, 1999; Morvai et al., 1997; Nobel, 2003, where this result is generalized and the history of its discovery is described. In particular, they found out that such a result was described by Bailey, 1976 in his unpublished thesis.) That is why it is difficult to use (2) for comparison of different predictors. On the other hand, it is shown in Ryabko $(1984,1988)$ that there exists a predictor $R$, such that the following average $t^{-1} \sum_{i=1}^{t} \rho_{R, p}\left(x_{1} \cdots x_{t}\right)$ goes to 0 (with probability 1 ) for any stationary and ergodic source $p$, where $t$ goes to infinity. That is why we will focus our attention on such averages. First, we define for any predictor $\gamma$ the following probability distribution:

$$
\gamma\left(x_{1} \ldots x_{t}\right)=\prod_{i=1}^{t} \gamma\left(x_{i} \mid x_{1} \ldots x_{i-1}\right)
$$

For example, we obtain for the Laplace predictor $L$ that $L(0101)=\frac{1}{2} \frac{1}{3} \frac{1}{2} \frac{2}{5}=\frac{1}{30}$, see (1). Then, by analogy with (2) we will estimate the error by $\mathrm{K}-\mathrm{L}$ divergence and define

$$
\bar{\rho}_{\gamma, p}\left(x_{1} \ldots x_{t}\right)=t^{-1}\left(\log \left(p\left(x_{1} \ldots x_{t}\right) / \gamma\left(x_{1} \ldots x_{t}\right)\right)\right)
$$

23 and

$$
\bar{\rho}_{t}(\gamma, p)=t^{-1} \sum_{x_{1} \ldots x_{t} \in A^{t}} p\left(x_{1} \ldots x_{t}\right) \log \left(p\left(x_{1} \ldots x_{t}\right) / \gamma\left(x_{1} \ldots x_{t}\right)\right) .
$$

For example, from those definitions and (3) we obtain the following bound for the Laplace predictor $L$ and any Bernoulli source $p: \bar{\rho}_{t}(L, p)<((|A|-1) \log t+c) / t$, where $c$ is a constant.

The universal predictors will play a key rule in the tests suggested below. By definition, a predictor $\gamma$ is called universal (in average) for a class of sources $M$, if for any $p \in M$ the error $\bar{\rho}_{t}(\gamma, p)$ goes to 0 , when $t$ goes to infinity. A predictor $\gamma$ is called universal with probability 1 , if the error $\bar{\rho}_{\gamma, p}\left(x_{1} \ldots x_{t}\right)$ goes to 0 not only in average, but for almost all sequences $x_{1} x_{2} \ldots$. In short, we will say that the predictor (or probability distribution) $\gamma$ is universal, if $\lim _{t \rightarrow \infty} \bar{\rho}_{\gamma, p}\left(x_{1} \ldots x_{t}\right)=0$ is valid with probability 1 for any stationary and ergodic source $p$ (i.e. for any $p \in M_{\infty}(A)$ ). Now there are quite many known universal predictors. One of the first such predictors has been described in Ryabko $(1984,1988)$. 


\subsection{Universal coding}

This short subparagraph is intended to give some explanation about why and how methods of data compression can be used for testing of independence. The point is that the prediction problem is deeply connected with the theory of universal coding. Moreover, practically used data compression methods (or so-called archivers) can be directly applied for testing.

Let us give some definitions. Let, as before, $A$ be a finite alphabet and, by definition,

$7 A^{*}=\bigcup_{n=1}^{\infty} A^{n}$ and $A^{\infty}$ is the set of all infinite words $x_{1} x_{2} \ldots$ over the alphabet $A$. A data compression method (or code) $\varphi$ is defined as a set of mappings $\varphi_{n}$ such that $\varphi_{n}: A^{n} \rightarrow$

$9\{0,1\}^{*}, n=1,2, \ldots$ and for each pair of different words $x, y \in A^{n} \varphi_{n}(x) \neq \varphi_{n}(y)$. Informally, it means that the code $\varphi$ can be applied for compression of each message of any

11 length $n, n>0$, over alphabet $A$ and the message can be decoded if its code is known. Further, it is required that each sequence $\varphi_{n}\left(x_{1}\right) \varphi_{n}\left(x_{2}\right) \ldots \varphi_{n}\left(x_{r}\right), r \geqslant 1$, of encoded words from the set $A^{n}, n \geqslant 1$, can be uniquely decoded into $x_{1} x_{2} \ldots x_{r}$. Such codes are called uniquely decodable. For example, let $A=\{a, b\}$, the code $\psi_{1}(a)=0, \psi_{1}(b)=00$, obviously, is not uniquely decodable. (Indeed, the word 000 can be decoded in both $a b$ and $b a$.) It is well known that if a code $\varphi$ is uniquely decodable then the lengths of the codewords satisfy the following inequality (the Kraft inequality):

$$
\sum_{u \in A^{n}} 2^{-\left|\varphi_{n}(u)\right|} \leqslant 1,
$$

see, for example, Gallager (1968). It will be convenient to reformulate this property as follows:

21 Claim 1. Let $\varphi$ be a uniquely decodable code over an alphabet $A$. Then for any integer $n$ there exists a measure $\mu_{\varphi}$ on $A^{n}$ such that

$$
-\log \mu_{\varphi}(u) \leqslant|\varphi(u)|
$$

for any $u$ from $A^{n}$. (Obviously, it is true for the measure $\mu_{\varphi}(u)=2^{-|\varphi(u)|} / \Sigma_{u \in A^{n}} 2^{-|\varphi(u)|}$.) It is well known that sequences $x_{1} \ldots x_{t}$, generated by a stationary and ergodic source $p$, can be "compressed" till the length $-\log p\left(x_{1} \ldots x_{t}\right)$ bits. There exist so-called universal codes, which, in a certain sense, are the best "compressors" for all stationary and ergodic sources. The formal definition is as follows: a code $\varphi$ is universal if for any stationary and ergodic source $p$

$$
\lim _{t \rightarrow \infty} t^{-1}\left(-\log p\left(x_{1} \ldots x_{t}\right)-\left|\varphi\left(x_{1} \ldots x_{t}\right)\right|\right)=0
$$

31 with probability 1. So, informally speaking, the universal codes estimate the probability characteristics of the source $p$ and use them for efficient "compression".

\section{The tests}

In this paragraph we describe the suggested tests. First, we give some definitions. Let $v$ be a word $v=v_{1} \ldots v_{k}, k \leqslant t, v_{i} \in A$. Denote the rate of a word $v$ occurring in the 
1 sequence $x_{1} x_{2} \ldots x_{k}, x_{2} x_{3} \ldots x_{k+1}, x_{3} x_{4} \ldots x_{k+2}, \ldots, x_{t-k+1} \ldots x_{t}$ by $v^{t}(v)$. For example, if $x_{1} \ldots x_{t}=000100$ and $v=00$, then $v^{6}(00)=3$. Now we define for any $k \geqslant 0$ the so-called

3 empirical Shannon entropy of order $k$ as follows:

$$
h_{k}^{*}\left(x_{1} \ldots x_{t}\right)=-\frac{1}{(t-k)} \sum_{v \in A^{k}} \bar{v}^{t}(v) \sum_{a \in A}\left(v^{t}(v a) / \bar{v}^{t}(v)\right) \log \left(v^{t}(v a) / \bar{v}^{t}(v)\right),
$$

5 where $k<t$ and $\bar{v}^{t}(v)=\sum_{a \in A} v^{t}(v a)$. In particular, if $k=0$, we obtain

$$
h_{0}^{*}\left(x_{1} \ldots x_{t}\right)=-\frac{1}{t} \sum_{a \in A} v^{t}(a) \log \left(v^{t}(a) / t\right) .
$$

$7 \quad$ The suggested test is as follows.

Let $\gamma$ be any probability distribution over $A^{t}$. The hypothesis $\mathrm{H}_{0}$ is accepted if

$$
(t-m) h_{m}^{*}\left(x_{1} \ldots x_{t}\right)-\log \left(1 / \gamma\left(x_{1} \ldots x_{t}\right)\right) \leqslant \log (1 / \alpha),
$$

where $0<\alpha<1$. Otherwise, $\mathrm{H}_{0}$ is rejected. We denote this test by $\Upsilon_{\alpha, \gamma, m}^{t}$.

Theorem. (i) For any predictor (or measure) $\gamma$ the Type I error of the test $\Upsilon_{\alpha, \gamma, m}^{t}$ is less than or equal to $\alpha, \alpha \in(0,1)$.

(ii) If $\gamma$ is a universal predictor (measure) (i.e., by definition, for any $p \in M_{\infty}(A)$

$$
\lim _{t \rightarrow \infty} t^{-1}\left(-\log p\left(x_{1} \ldots x_{t}\right)-\log \left(1 / \gamma\left(x_{1} \ldots x_{t}\right)\right)\right)=0
$$

with probability 1), then the Type II error goes to 0, when t goes to infinity.

The proof is given in Appendix.

Comment. Let $\varphi$ be a uniquely decodable code (or a data compression method). Define the test $\hat{\Upsilon}_{\alpha, \varphi, m}^{t}$ as follows: The hypothesis $\mathrm{H}_{0}$ is accepted if

$$
(t-m) h_{m}^{*}\left(x_{1} \ldots x_{t}\right)-\left|\varphi\left(x_{1} \ldots x_{t}\right)\right| \leqslant \log (1 / \alpha),
$$

where $\alpha \in(0,1)$. Otherwise, $\mathrm{H}_{0}$ is rejected.

We immediately obtain from the Theorem 1 and the Claim 1 the following statement.

Claim 2. (i) For any uniquely decodable code $\varphi$ the Type I error of the test $\hat{\Upsilon}_{\alpha, \varphi, m}^{t}$ is less than or equal to $\alpha, \alpha \in(0,1)$.

(ii) If $\varphi$ is a universal code, then the Type II error goes to 0 , where $t$ goes to infinity.

\section{Conclusion}

The tests described above can be based on known universal codes (or so-called archivers) which are widely used for text compression everywhere. It is important to note that, on the one hand, the universal codes and archivers are based on results of Information Theory, the theory of algorithms and some other branches of mathematics; see, for example, Rissanen 
1 (1984), Kieffer (1998), Kieffer and Yang (2000), Effros et al. (2002). On the other hand, the archivers have shown high efficiency in practice as compressors of texts, DNA sequences and many other types of real data. In fact, the archivers can find many kinds of latent regularities, which is why they look like a promising tool for independence testing and its generalizations.

The natural question is the possibility of generalizing the suggested tests for the case of an infinite source alphabet $A$ (say, $A$ is a metric space.) Apparently, such a generalization can be done for the case of independence testing, if we will use a known technique of partitioning; see Darbellay and Vajda $(1998,1999)$. But we do not know how to generalize the suggested tests for the case where $\mathrm{H}_{0}$ is that the source is Markovian. The point is that the partitioning can increase the source order. For example, even if the alphabet $A$ contains three letters and we combine two of them in one subset (i.e. a new letter) the order of the obtained source can increase till infinity. Hence, the generalization to Markov sources with infinite alphabet can be considered as an open problem.

\section{Appendix}

Proof of Theorem. First we show that for any Bernoulli source $\tau^{*}$ and any word $x_{1} \ldots x_{t} \in$ $A^{t}, t>1$, the following inequality is valid:

$$
\tau^{*}\left(x_{1} \ldots x_{t}\right)=\prod_{a \in A} \tau(a)^{v^{t}(a)} \leqslant \prod_{a \in A}\left(n u^{t}(a) / t\right)^{v^{t}(a)} .
$$

Indeed, the equality is true, because $\tau^{*}$ is a Bernoulli measure. The inequality follows from the well-known inequality $\sum_{a \in A} p(a) \log (p(a) / q(a)) \geqslant 0$, for $\mathrm{K}-\mathrm{L}$ divergence, which is true for any distributions $p$ and $q$ (see, for example, Gallager (1968)). So, if $p(a)=v^{t}(a) / t$ and $q(a)=\tau^{*}(a)$, then

$$
\sum_{a \in A} \frac{v^{t}(a)}{t} \log \frac{\left(v^{t}(a) / t\right)}{\tau(a)} \geqslant 0 .
$$

From the last inequality we obtain (11).

Let now $\tau$ belong to $M_{m}(A), m>0$. We will prove that for any $x_{1} \ldots x_{t}$

$$
\tau\left(x_{1} \ldots x_{t}\right) \leqslant \prod_{u \in A^{m}} \prod_{a \in A}\left(v^{t}(u a) / \bar{v}^{t}(u)\right)^{v^{t}(u a)} .
$$

Indeed, we can present $\tau\left(x_{1} \ldots x_{t}\right)$ as

$$
\tau\left(x_{1} \ldots x_{t}\right)=\tau_{\infty}\left(x_{1} \ldots x_{m}\right) \prod_{u \in A^{m}} \prod_{a \in A} \tau(a / u)^{v^{t}(u a)},
$$

29 where $\tau_{\infty}\left(x_{1} \ldots x_{m}\right)$ is the limit probability of the word $x_{1} \ldots x_{m}$. From the last equality we can see that

$$
\tau\left(x_{1} \ldots x_{t}\right) \leqslant \prod_{u \in A^{m}} \prod_{a \in A} \tau(a / u)^{v^{t}(u a)} .
$$


1 Taking into account inequality (11), we obtain

$$
\prod_{a \in A} \tau(a / u)^{v^{t}(u a)} \leqslant \prod_{a \in A}\left(v^{t}(u a) / \bar{v}^{t}(u)\right)^{v^{t}(u a)}
$$

3 for any word $u$. So, from the last two inequalities we obtain (12).

It will be convenient to define an auxiliary measure on $A^{t}$ as follows:

$$
\pi_{m}\left(x_{1} \ldots x_{t}\right)=\Delta 2^{-(t-m)} h_{m}^{*}\left(x_{1} \ldots x_{t}\right),
$$

where $x_{1} \ldots x_{t} \in A^{t}$ and $\Delta=\left(\sum_{x_{1} \ldots x_{t} \in A^{t}} 2^{-(t-m) h_{m}^{*}\left(x_{1} \ldots x_{t}\right)}\right)^{-1}$. If we take into account that $2^{-(t-m)} h_{m}^{*}\left(x_{1} \ldots x_{t}\right)=\prod_{u \in A^{m}} \prod_{a \in A}\left(v^{t}(u a) / \bar{v}^{t}(u)\right)^{v^{t}(u a)}$, we can see from (12) and (13) that, for any measure $\tau \in M_{m}(A)$ and any $x_{1} \ldots x_{t} \in A^{t}$,

$\tau\left(x_{1} \ldots x_{t}\right) \leqslant \pi_{m}\left(x_{1} \ldots x_{t}\right) / \Delta$.

Let us denote the critical set of the test $\Upsilon_{\alpha, \gamma, m}^{t}$ as $C_{\alpha}$ i.e., by definition,

$$
C_{\alpha}=\left\{x_{1} \ldots x_{t}:(t-m) h_{m}^{*}\left(x_{1} \ldots x_{t}\right)-\log \left(1 / \gamma\left(x_{1} \ldots x_{t}\right)\right)>\log (1 / \alpha)\right\} .
$$

From (14) and this definition we can see that for any measure $\tau \in M_{m}(A)$

$$
\tau\left(C_{\alpha}\right) \leqslant \pi_{m}\left(C_{\alpha}\right) / \Delta \text {. }
$$

From definitions (15) and (13) we obtain

$$
\begin{aligned}
C_{\alpha} & =\left\{x_{1} \ldots x_{t}: 2^{(t-m) h_{m}^{*}\left(x_{1} \ldots x_{t}\right)}>\left(\alpha \gamma\left(x_{1} \ldots x_{t}\right)\right)^{-1}\right\} \\
& =\left\{x_{1} \ldots x_{t}:\left(\pi_{m}\left(x_{1} \ldots x_{t}\right) / \Delta\right)^{-1}>\left(\alpha \gamma\left(x_{1} \ldots x_{t}\right)\right)^{-1}\right\} .
\end{aligned}
$$

Finally,

$$
C_{\alpha}=\left\{x_{1} \ldots x_{t}: \gamma\left(x_{1} \ldots x_{t}\right)>\pi_{m}\left(x_{1} \ldots x_{t}\right) /(\alpha \Delta)\right\} .
$$

The following inequalities and equalities are valid:

$$
\begin{aligned}
1 & \geqslant \sum_{x_{1} \ldots x_{t} \in C_{\alpha}} \gamma\left(x_{1} \ldots x_{t}\right) \geqslant \sum_{x_{1} \ldots x_{t} \in C_{\alpha}} \pi_{m}\left(x_{1} \ldots x_{t}\right) /(\alpha \Delta) \\
& =\pi_{m}\left(C_{\alpha}\right) /(\alpha \Delta) \geqslant \tau\left(C_{\alpha}\right) \Delta /(\alpha \Delta)=\tau\left(C_{\alpha}\right) / \alpha .
\end{aligned}
$$

(Here both equalities and the first inequality are obvious, the second inequality and the third one follow from (17) and (16), correspondingly.) So, we obtain that $\tau\left(C_{\alpha}\right) \leqslant \alpha$ for any measure $\tau \in M_{m}(A)$. Taking into account that $C_{\alpha}$ is the critical set of the test, we can see that the probability of the Type I error is not greater than $\alpha$. The first claim of the theorem is proven.

The proof of the second statement of the theorem will be based on some results of Information Theory. The $t$-order conditional Shannon entropy is defined as follows:

$$
h_{t}(p)=-\sum_{x_{1} \ldots x_{t} \in A^{t}} p\left(x_{1} \ldots x_{t}\right) \sum_{a \in A} p\left(a / x_{1} \ldots x_{t}\right) \log p\left(a / x_{1} \ldots x_{t}\right)
$$

where $p \in M_{\infty}(A)$. It is known that for any $p \in M_{\infty}(A)$ firstly, $\log |A| \geqslant$ $h_{0}(p) \geqslant h_{1}(p) \geqslant \ldots$, secondly, the following limit Shannon entropy $h_{\infty}(p)=\lim _{t \rightarrow \infty} h_{t}(p)$ 
1 exists, thirdly, $\lim _{t \rightarrow \infty}-t^{-1} \log p\left(x_{1} \ldots x_{t}\right)=h_{\infty}(p)$ with probability 1 and, finally, $h_{m}(p)$ is strictly greater than $h_{\infty}(p)$, if the memory of $p$ is larger $m$, (i.e. $p \in M_{\infty}(A) \backslash M_{m}(A)$ ), see, for example, Billingsley (1965), Gallager (1968).

Taking into account the definition of the universal predictor (see (9)), we obtain from the above described properties of the entropy that

$$
\lim _{t \rightarrow \infty}-t^{-1} \log \gamma\left(x_{1} \ldots x_{t}\right)=h_{\infty}(p)
$$

with probability 1 . It can be seen that $h_{m}^{*}(7)$ is a consistent estimate for the $m$ - order Shannon entropy (18), i.e. $\lim _{t \rightarrow \infty} h_{m}^{*}\left(x_{1} \ldots x_{t}\right)=h_{m}(p)$ with probability 1; see Billingsley

9 (1965), Gallager (1968). Having taken into account that $h_{m}(p)>h_{\infty}(p)$ and (19) we obtain from the last equality that $\lim _{t \rightarrow \infty}\left((t-m) h_{m}^{*}\left(x_{1} \ldots x_{t}\right)-\log \left(1 / \gamma\left(x_{1} \ldots x_{t}\right)\right)\right)=\infty$. This proves the second statement of the theorem.

\section{References}

Algoet, P., 1999. Universal schemes for learning the best nonlinear predictor given the infinite past and side information. IEEE Trans. Inform. Theory 45, 1165-1185.

Bailey, D.H., 1976. Sequential schemes for classifying and predicting ergodic processes. Ph.D. Dissertation, Stanford University.

17 Billingsley, P., 1965. Ergodic Theory and Information. Wiley, New York.

Blum, J.R., Kiefer, J., Rosenblatt, M., 1961. Distribution free tests of independence based on the sample distribution function. Ann. Math. Statist. 32, 485-498.

Csiszár, I., Shields, P., 2000. The consistency of the BIC Markov order estimation. Ann. Statist. 6, 1601-1619.

Darbellay, G.A., Vajda, I., 1998. Entropy expressions for multivariate continuous distributions. Research Report No. 1920, UTIA, Academy of Science, Prague (library@utia.cas.cz).

23 Darbellay, G.A., Vajda, I., 1999. Estimation of the mutual information with data-dependent partitions. IEEE Trans. Inform. Theory 48 (5), 1061-1081.

Effros, M., Visweswariah, K., Kulkarni, S.R., Verdu, S., 2002. Universal lossless source coding with the Burrows Wheeler transform. IEEE Trans. Inform. Theory 48 (5), 1061-1081.

27 Feller, W., 1970. An Introduction to Probability Theory and its Applications, vol. 1. Wiley, New York.

Gallager, R.G., 1968. Information Theory and Reliable Communication. Wiley, New York.

Ghoudi, K., Kulperger, R.J., Remillard, B., 2001. A nonparametric test of serial independence for time series and residuals. J. Multivariate Anal. 79 (2), 191-218.

31 Hoeffding, W., 1948. A nonparametric test of independence. Ann. Math. Statist. 19, 546-557.

Kendall, M.G., Stuart, A., 1961. The Advanced Theory of Statistics, vol. 2. Inference and Relationship. Charles Griffin, London.

Kieffer, J., 1998. Prediction and Information Theory, Preprint (available at $\mathrm{ftp} / / /$ oz.ee.umn.edu/users/kieffer/papers/prediction.pdf/).

Kieffer, J.C., Yang, E.H., 2000. Grammar-based codes: a new class of universal lossless source codes. IEEE Trans. Inform. Theory 46 (3), 737-754.

Morvai, G., Yakowitz, S.J., Algoet, P.H., 1997. Weakly convergent nonparametric forecasting of stationary time series. IEEE Trans. Inform. Theory 43, 483-498.

Nobel, A.B., 2003. On optimal sequential prediction. IEEE Trans. Inform. Theory 49 (1), 83-98.

41 Rissanen, J., 1984. Universal coding, information, prediction, and estimation. IEEE Trans. Inform. Theory 30 (4), 629-636.

43 Ryabko, B., Monarev, V., 2005. Using information theory approach to randomness testing. J. Statist. Plann. Inference 133 (1), 95-110.

45 Ryabko, B., Topsoe, F., 2002. On asymptotically optimal methods of prediction and adaptive coding for Markov sources. J. Complexity 18 (1), 224-241. 
1 Ryabko, B.Ya., 1984. Twice-universal coding. Problems Inform. Transmission 20 (3),

Ryabko, B.Ya., 1988. Prediction of random sequences and universal coding. Problems Inform. Transmission 24 (2), 87-96.

Ryabko, B.Ya., 1990. A fast adaptive coding algorithm. Problems Inform. Transmission 26 (4), 305-317. 